\title{
水解酶的非专一性催化在有机合成中的应用
}

\author{
葛 新 ${ }^{a}$ 赖依峰 ${ }^{a, b}$ 陈新志*, $a$ \\ ( ${ }^{a}$ 浙江大学生物质化工教育部重点实验室 杭州 310027) \\ ( ${ }^{b}$ 浙江科技学院生物与化学工程学院 杭州 310012)
}

\begin{abstract}
摘要 介绍了水解酶非专一性催化的机理, 对水解酶的非专一性催化在单元、两步和一锅三组分的有机反应中, 特别 是加成反应、Aldol 反应和 Knoevenagel 反应中的研究与应用进行了总结和概述.

关键词 水解酶; 非专一性; Michael 加成; Aldol 反应; 一锅三组分

\section{Application of Hydrolase Catalytic Promiscuity in Organic Synthesis}

\author{
Ge, Xin ${ }^{a} \quad$ Lai, Yifeng ${ }^{a, b} \quad$ Chen, Xinzhi ${ }^{*, a}$
}

( ${ }^{a}$ Key Laboratory of Biomass Chemical Engineering of Ministry of Education, Zhejiang University, Hangzhou 310027)

( ${ }^{b}$ Zhejiang University of Science and Technology, School of Biological and Chemical Engineering, Hangzhou 310012)

\begin{abstract}
This review introduces the mechanism of hydrolase promiscuity. Several applications of hydrolase catalytic promiscuity in unit reaction, two-step reaction and three-component reaction are summarized, especially for addition, aldol reaction, and Knoevenagel reaction.

Keywords hydrolase; promiscuity; Michael addition; aldol reaction; three-component reaction
\end{abstract}

酶一直被认为是一种高效、专一、具有很强的立体 选择性的生物催化剂, 但是近年来研究发现许多酶除了 具有天然催化功能外, 还能够催化其他反应, 即酶的催 化多功能性, 也称为酶的催化非专一性 ${ }^{[1]}$. 在生命起源 时, 酶的非专一性催化使酶扮演着 “多面手” 的角色, 催 化得到多种化合物, 使得自然界物质日益丰富 ${ }^{[2]}$. 通过 不断进化, 酶表现出很强的专一性, 但是由于酶的复杂 结构和进化过程中的不完美控制, 很多酶仍旧残留着非 专一性催化的功能, 只是催化效率较低. 将酶的非专一 性催化和有机合成相结合, 可以拓展有机合成的方法学 和酶化学的新领域, 在近年来受到研究者的普遍关注. 水解酶, 作为自然界中应用最广泛的一大类酶, 目前其 非专一性催化已被应用于多种有机反应中.

水解酶的非专一性催化在有机合成中的研究主要 集中于 $\mathrm{C}-\mathrm{C}$ 键的反应, 如加成反应、Aldol 反应等. 目 前对于水解酶的非专一性催化单元反应的基础研究主 要集中于不同酶和反应的大量篮选. 考虑其经济价值,
有很多研究者将目光转向单酶或多酶催化的多步一锅 合成，其可以缩减多步反应中的分离纯化步骤. 通常在 多酶催化体系中，由于不同酶的催化活性都需要特定的 $\mathrm{pH}$ 值和温度等影响条件, 因此每步反应都需要进行严 格控制, 操作过程相对繁琐, 而且一些酶会因为缺乏特 定的反应条件而失活. 单酶催化一锅法则能克服多酶催 化体系的这些缺点, 利用单酶具有的催化多种反应的能 力, 集成多步催化反应在一个体系中完成. 其过程中无 需分离和提纯, 大大简化了反应步骤与操作.

本文将基于水解酶非专一性催化在有机合成中的 潜在价值和优势, 主要对水解酶的非专一性催化机理和 其在单元反应、两步和一锅三组分有机反应中的研究与 应用进展进行总结和概述.

\section{1 水解酶的非专一性催化机理}

水解酶是催化水解反应的一类酶的总称, 用水作为 被转移基团的受体，应用非常广泛. 水解酶中常见的有

\footnotetext{
* E-mail: xzchen@zju.edu.cn

Received January 4, 2013; revised February 4, 2013; published online February 18, 2013

Project supported by the Key Innovation Group of Zhejiang Province (No. 2009R50002).

浙江省重点科技创新团队(No. 2009R50002)资助项目.
} 
蛋白酶、淀粉酶和脂肪酶等, 其中对脂肪酶和蛋白酶的 催化非专一性研究较多. 水解酶通常是催化酯键的形成 或断裂, 但近来研究发现水解酶还具有催化 Aldol 反应、 Michael 加成、Knovenegal 等反应的活性.

水解酶的非专一性催化理论研究主要集中在 $\alpha / \beta$ 水 解酶超家族酶类, 这类酶含有一个 “亲核类氨基酸-His酸类氨基酸” 催化三联体, 这类催化三联体可以形成高 效的针对不同化合物或具有不同物理化学性质的底物 的运转机制, 以适应不同的生理环境. 在水解酶反应机 制中, 氧离子穴是一个非常重要的特征. 在以酯为底物 时, 氧离子穴可以使羰基双键极化, 极化的羰基碳易受 丝氨酸活性位点的亲核攻击使得酯键水解. 目前研究认 为, 水解酶的催化非专一性的活性位点还是位于酶的原 始活性位点上, 主要差异是底物的定位不同. 而氧离子 洞在酶的非专一性催化中仍然发挥着激活羰基基团的 作用，是水解酶催化非水解反应的基础.

南极假丝酵母脂肪酶(CALB) 是应用于研究酶的非 专一性催化较多的一种酶, 它的非专一性催化活性由 Ser-His-Asp 催化三联体介导, 运用其氧离子穴, CALB 同样能够催化许多不同的 $\mathrm{C}-\mathrm{C}$ 键形成反应 ${ }^{[3]}$. 其催化 Michael 加成(1)和 aldol 反应(2)的机理类似: 通过形成烯 醇式中间体, 与酶上活性位点的氧负离子空洞和催化三 联体作用进行反应, 活性位点组氨酸作为碱基吸引一个 质子, 最后和第二底物形成 $\mathrm{C}-\mathrm{C}$ 键. Berglund 等 ${ }^{[4]}$ 首先 将天然酶用甲基-对硝基苯-正己基磷酸酯共价抑制而 完全失去活性, 这证明活性中心对于催化这个反应而言 是必须的; 然后比较 CALB Ser105Ala 和 CALB 的催化 Michael 加成和 aldol 反应的酶活, 发现突变后的酶活与 野生酶的相比提高了很多. 后来也比较了 CALB 突变前 后, 在水介质中催化 Michael 加成和水解的酶活, 发现

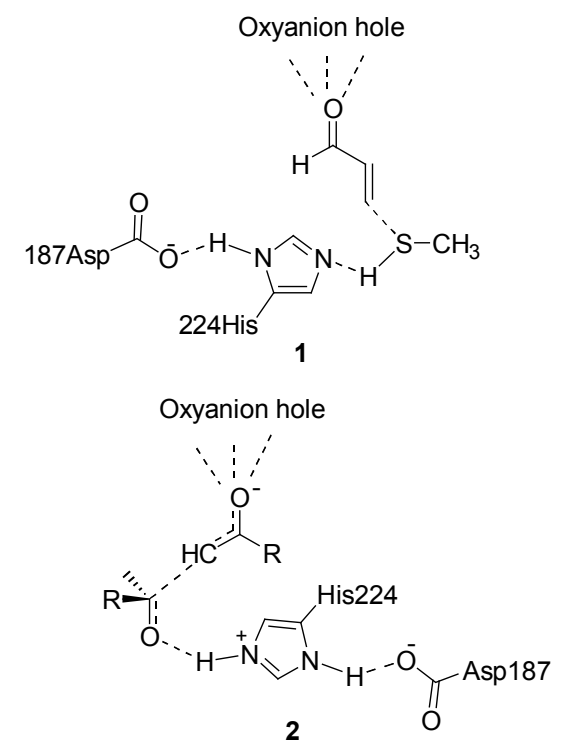

突变后水解能力降低了 1000 倍, 但是 Michael 加成的酶 活增加了 100 倍 $^{[5]}$. 由这些推测可能因为丝氨酸通过与 His-Asp 形成氢键, 从而降低了 His-Asp 夺质子的能力, 结合于量子化学模拟, 确认反应是发生在酶的活性中 心, 但是这个结论仍需要更多的实验证明. 目前大部分 水解酶的非专一性催化有机合成的反应机理都是依此 推测的.

\section{2 水解酶非专一性催化在有机合成中的应用}

\section{1 单元反应}

水解酶的非专一性催化在有机合成中的研究主要 集中于 $\mathrm{C}-\mathrm{C}$ 键的反应，包括加成反应(主要是 Michael 加成)、Aldol 反应和 Knovenegal 反应等(表 1$)^{[6 \sim 19]}$. 脂肪 酶和蛋白酶在水解酶的非专一性催化中研究的较多.

\section{1 .1 加成反应}

加成反应是有机合成反应中较为重要的反应, 研究 发现一些水解酶在一定的条件下具有催化加成反应的 的活性，其中 Michael 加成是最早同时也是最多的用于 研究水解酶非专一性催化的反应.

最早 1986 年 Kitazume 等 ${ }^{[6]}$ 研究氟化物 4 的不对称 Michael 加成反应(Eq. 1)时发现, 在 $\mathrm{pH}=8.0$ 的缓冲溶液 中，以 2-三氟甲基丙烯酸(3)为原料，猪肝酯酶(Pig liver esterase)， $\alpha$-胰凝乳蛋白酶( $\alpha$-Chymotrypsin)和柱状假丝 酵母脂肪酶(Candida cylindracea Lipase)可以成功催化 该反应(表 2).

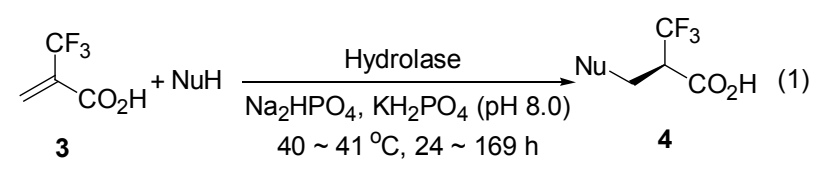

1984 年 Klibanov 等 ${ }^{[20]}$ 在有机介质中成功实现酶催 化有机合成, 这彻底改变了酶促合成只在水溶液中进行 的传统性认识, 拓宽了酶对底物的催化应用范围, 使许 多不能在水中进行的酶催化反应能够在有机溶剂中进 行 $^{[21]}$. 有机溶剂因为种类多、理化性质选择的余地比较 大和容易操作等优点而受到了广泛的重视. 2004 年林贤 福课题组 ${ }^{[7]}$ 首先在有机介质 DMSO 和吡啶中使用枯草 杆菌碱性蛋白酶(Alkaline Protease from Bacillus subtilis) 分别催化咪唑及其衍生物 5、嘧啶及其衍生物和丙烯酸 酯 6 发生 Michael 加成(Eq. 2), 取得了很好的效果. 之后 2006 年该课题 ${ }^{[22]}$ 系统研究了在有机介质中用不同的水 解酶催化咪唑及其衍生物与丙烯酸酯的 $\mathrm{C}-\mathrm{N}$ 键 Michael 加成反应. 发现有 9 种水解酶可以催化 Michael 加成, 由于这些水解酶都属于丝氨酸水解酶家族, 推测 水解酶催化 Michael 加成可能发生在水解酶的催化活性 中心. 该课题组 ${ }^{[23]}$ 继续研究发现, 具有锌活性位点的酰 
表 1 水解酶的非专一性催化单元反应

Table 1 The hydrolase-promiscuous catalytic unit reaction

\begin{tabular}{|c|c|c|c|c|c|}
\hline Reaction & Substrate 1 & Substrate 2 & Hydrolase & Yield & References \\
\hline \multirow{9}{*}{ Michael addition } & & $\mathrm{H}_{2} \mathrm{O}$ & $\begin{array}{l}\text { Candida cylindracea } \\
\text { lipase }\end{array}$ & $48 \%(34 \mathrm{~h})$, ee $70 \%$ & \\
\hline & $\mathrm{CO}_{2} \mathrm{H}$ & $\mathrm{Et}_{2} \mathrm{NH}$ & $\begin{array}{l}\text { Candida cylindracea } \\
\text { lipase }\end{array}$ & $47 \%$ (92 h), ee 71\% & [6] \\
\hline & & $\mathrm{PhNH}_{2}$ & $\begin{array}{l}\text { Candida cylindracea } \\
\text { lipase }\end{array}$ & $76 \%$ (40 h), eе $39 \%$ & \\
\hline & & & $\begin{array}{l}\text { Alkaline protease from } \\
\text { Bacillus subtilis }\end{array}$ & $76 \%(96 \mathrm{~h})$ & [7] \\
\hline & & & Lipozyme RM IM & $92 \%(1 \mathrm{~h})$ & \\
\hline & & & Lipozyme TL IM & $85 \%(1.2 \mathrm{~h})$ & [8] \\
\hline & & & Novozyme 435 & $70 \%(1.2 \mathrm{~h})$ & \\
\hline & & & $D$-Aminoacylase & $94 \%(16 \mathrm{~h})$ & [9] \\
\hline & & $\mathrm{NO}_{2}$ & CALB & $99 \%(20 \mathrm{~h})$ & {$[10]$} \\
\hline \multirow{5}{*}{ Aldol reaction } & & & PPL & $96.4 \%(144 \mathrm{~h})$, ee $14.7 \%$ & [11] \\
\hline & & & Pepsin & $22 \%(24 \mathrm{~h})$, ee $43 \%$ & [12] \\
\hline & & & $\begin{array}{l}\text { Alkaline protease from } \\
\text { Bacillus licheniformis }\end{array}$ & $57 \%$ (47 h), eе $49 \%$ & [13] \\
\hline & & & $\begin{array}{l}\text { Acidic protease from } \\
\text { Aspergillus usamii }\end{array}$ & $63 \%(144 \mathrm{~h})$, ee $82 \%$ & [14] \\
\hline & & & Chymopapain & $69 \%(131 \mathrm{~h})$, ee $78 \%$ & {$[15]$} \\
\hline \multirow{2}{*}{$\begin{array}{l}\text { Knoevenagel } \\
\text { reaction }\end{array}$} & & & PPL & $85 \%(12 \mathrm{~h})$ & [16] \\
\hline & & & Papain & $59 \%(120 \mathrm{~h})$ & {$[17]$} \\
\hline \multirow{3}{*}{ Henry reaction } & & $\mathrm{H}_{3} \mathrm{C}-\mathrm{NO}_{2}$ & $\begin{array}{l}\text { Immobilized lipase from } \\
\text { Thermomyces lanuginosus }\end{array}$ & $64 \%(48 \mathrm{~h})$ & [18] \\
\hline & & & $D$-aminoacylase & $83 \%(2 \mathrm{~h})$ & \multirow[b]{2}{*}{ [19] } \\
\hline & $\mathrm{NO}_{2}$ & $\mathrm{NO}_{2}$ & $\begin{array}{l}\text { Amano lipase } \mathrm{M} \text { from } \\
\text { M. javanicus }\end{array}$ & $75 \%(2 \mathrm{~h})$ & \\
\hline
\end{tabular}

基转移酶在 DMSO 中也可以催化含氮的芳香杂环与 $\alpha, \beta$-不饱和羰基化合物的 Michal 加成反应，与具有丝氨 酸活性位点的水解酶相比具有更好的催化效果. 之后他 们又陆续报道了 $D$-氨基酰化氨基酸水解酶不仅催化嘌 呤衍生物和 $\alpha, \beta$-不饱和羰基化合物发生 Michael 反应,
产物的收率在 $80 \%$ 以上 ${ }^{[24]}$; 还可以在有机溶剂中催化 吡咯衍生物和乙烯酯类发生 Markovnikov 加成, 收率最 高至 $91 \%{ }^{[25]}$

2005 年 Gotor 等 ${ }^{[26]}$ 用脂肪酶 Chirazyme L-2、冻干 的 CALB 和 Novozyme 435 催化了肿胺 8 与丙烯腈 9 的 
表 2 水解酶催化的 Michael 加成反应

Table 2 Michael addition catalyzed by hydrolase

\begin{tabular}{lcccc}
\hline \multicolumn{1}{c}{ Enzyme } & $\mathrm{NuH}$ & Time/h & Yield/\% & ee/\% \\
\hline Candida cylindracea & $\mathrm{H}_{2} \mathrm{O}$ & 34 & 48 & 70 \\
Pig liver esterase & $\mathrm{H}_{2} \mathrm{O}$ & 52 & 54 & 60 \\
a-Chymotrypsin & $\mathrm{H}_{2} \mathrm{O}$ & 169 & 77 & 49 \\
Candida cylindracea & $\mathrm{EtOH}$ & 48 & - & - \\
Pig liver esterase & $\mathrm{PhOH}$ & 48 & - & - \\
Candida cylindracea & $\mathrm{Et}_{2} \mathrm{NH}$ & 92 & 47 & 71 \\
Pig liver esterase & $\mathrm{Et}_{2} \mathrm{NH}$ & 92 & 39 & 69 \\
Candida cylindracea & $\mathrm{PhNH}_{2}$ & 40 & 76 & 39 \\
Pig liver esterase & $\mathrm{PhSH}^{24}$ & 24 & 64 & 50 \\
\hline
\end{tabular}

$$
\begin{aligned}
\mathrm{R}^{1} & =\mathrm{R}^{2}=\mathrm{H}, \mathrm{R}^{3}=\mathrm{CH}_{3}, 76 \% \\
\mathrm{R}^{1} & =\mathrm{R}^{2}=\mathrm{H}, \mathrm{R}^{3}=\mathrm{CH}_{2} \mathrm{CH}_{3}, 65 \% \\
\mathrm{R}^{1} & =\mathrm{R}^{2}=\mathrm{H}, \mathrm{R}^{3}=\mathrm{CH}_{2} \mathrm{CH}_{2} \mathrm{CH}_{2} \mathrm{CH}_{3}, 62 \% \\
\mathrm{R}^{1} & =\mathrm{CH}_{3}, \mathrm{R}^{2}=\mathrm{NO}_{2}, \mathrm{R}^{3}=\mathrm{CH}_{3}, 75 \% \\
\mathrm{R}^{1} & =\mathrm{H}, \mathrm{R}^{2}=\mathrm{NO}_{2}, \mathrm{R}^{3}=\mathrm{CH}_{3}, 64 \%
\end{aligned}
$$

Michael 加成(Eq. 3), 并且提出了催化机理: 脂肪酶活性 位点的氧负离子穴极化了腈基, 从而活化丙烯腈, 催化 三联体中的 His-Asp 对转移了仲胺的质子, 最终质子转 移到 $\alpha$-碳上得到目标化合物(Scheme 1). Souza 等 ${ }^{[8]}$ 通过 使用不同的水解脂肪酶催化伯胺或者仲胺与丙烯腈的 Michael 加成反应, 发现所有的产物收率都可以达到 $60 \%$ 以上, 催化效率也很高, 并且推测脂肪酶催化伯胺 或者仲胺与丙烯腈的 Michael 加成反应的机理: 底物的 活化主要是通过氰基的极化来完成的.

$$
\overbrace{9}
$$

2005 年 Berglund 等 ${ }^{[3]}$ 通过量子化学模拟验证了这 个机理, 他们 ${ }^{[4 b]}$ 还报道了用 CALB Ser105Ala 在环己烷 中催化 1,3-羰基化合物 $\mathbf{1 1}$ 与 $\alpha, \beta$-不饱和羰基化合物 12 的 Michael 加成(Eq. 4). 林贤福等 ${ }^{[9]}$ 发现 11 种水解酶(枯 草芽孢杆菌碱性蛋白酶、米曲蛋白酶、猪胰脂肪酶和 $D$-氨基酰化氨基酸水解酶等)在 2-甲基-2-丁醇中也可以 催化该反应，收率最高达到 94\%。

2012 年 Madalinska 等 ${ }^{[27]}$ 报道了水解酶催化 $\alpha, \beta$-不 饱和亚硫酰基的 Michael 加成, 以苯硫酚和乙烯基亚硫 酰基苯为底物时, 反应仅在水解酶存在和乙醇溶剂中发

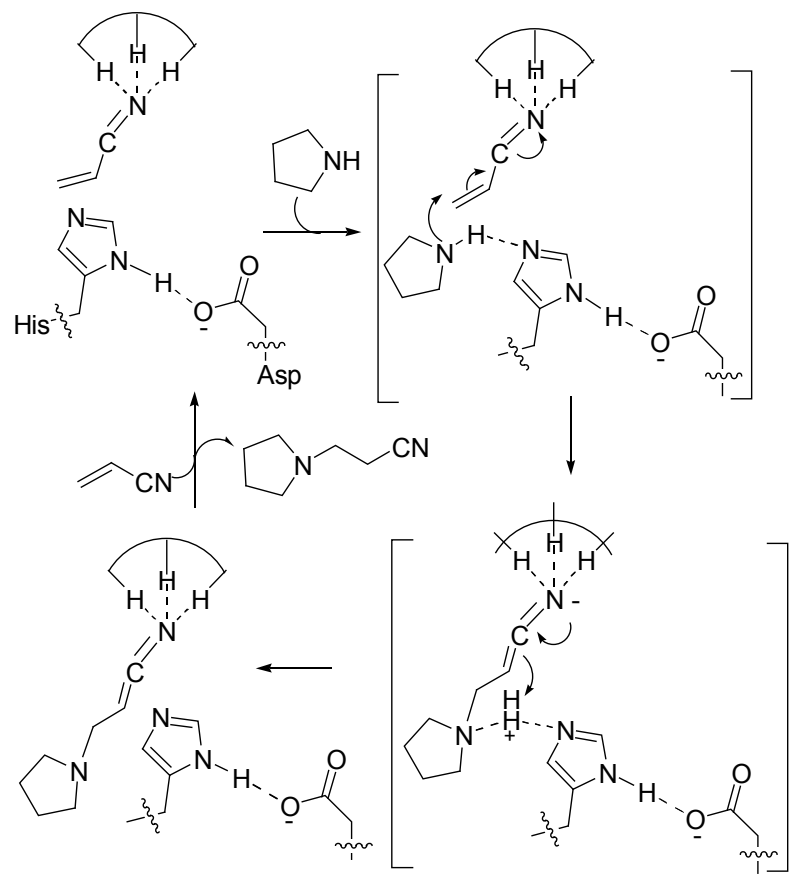

Scheme 1

$$
\begin{aligned}
& \mathrm{R}_{\mathrm{R}^{1}}=\mathrm{Me}, \mathrm{R}^{2}=\mathrm{H} ; \\
& \mathrm{R}^{1}=\mathrm{OMe}, \mathrm{R}^{2}=\mathrm{Me} ; \\
& \mathrm{R}^{1}=\mathrm{OMe}, \mathrm{R}^{2}=\mathrm{OMe}
\end{aligned}
$$

生，反应产物是外消旋，收率为 $20 \% \sim 35 \%$. 当使用更 强的 Michael 加成供体时，发现产物有立体选择性，该 研究证明该反应的机理不能用水解酶催化烯䣶和丙烯 腈的 Michael 加成反应机理来解释, 推测可能不是酶原 有的活性催化该反应，而是有了新的非专一性活性中 心.

\subsubsection{Aldol 反应}

Aldol 反应是有机化学中形成 $\mathrm{C}-\mathrm{C}$ 键的重要反应, 也是较早被应用于水解酶非专一性催化研究的反应. 水 解酶非专一性催化 Aldol 反应已经由较早的酶活很低、 没有对映选择性发展到最近的不对称合成.

2003 年 Berglund 等 ${ }^{[4 a]}$ 首次发现 CALB 具有催化 aldol 反应的活性, 然后用 CALB Ser105Ala 来催化着醛 缩合反应(Eq. 5)发现具有更高的催化活性. 反应的机理 是通过形成烯醇式中间体，与酶上活性位点的氧负离子 空洞和催化三联体作用进行反应.但是 CALB 催化 aldol 反应没有对映选择性，反应时间过长，因此应用价值不 $大^{[28]}$. 


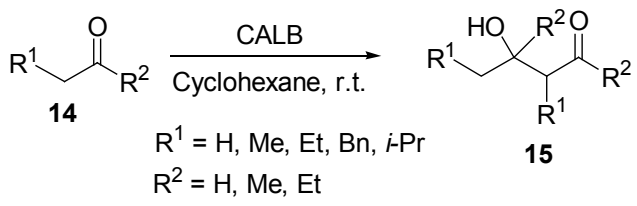

2008 年余孝其等 ${ }^{[11]}$ 首先发现了一些天然水解酶(猪 胰脂肪酶 PPL、爪哇毛霉菌脂肪酶 MJL、灭黑毛霉菌脂 肪酶 MML, CALB) 能够催化对硝基苯甲醛 $\mathbf{1 5}$ 与丙䤊 16 的不对称 aldol 反应(Eq. 6), ee 值为 $43.6 \%$ (表 3). 在该反 应中水起到非常重要的作用, 当整个反应体系是无水的 有机溶剂时, 酶几乎没有催化活性, 体系的含水量增加 到 $20 \%$ 时, 酶的催化活性达到最高. 在 2010 年该课题 组 ${ }^{[12]}$ 发现蛋白酶也可以在水介质中催化芳香醛与丙酮 的不对称 aldol 反应，收率最高达到 69\%, ee 值为 44\%.

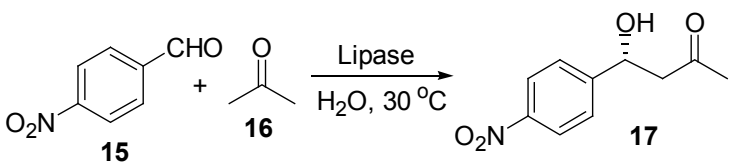

表 3 水解酶催化 aldol 反应

Table 3 Aldol reaction catalyzed by lipase

\begin{tabular}{ccccc}
\hline Entry & Catalyst & Reaction time/h & Yield/\% & $e e / \%$ \\
\hline 1 & PPL & 144 & 96.4 & 14.7 \\
2 & PPL & 72 & 11.7 & 43.6 \\
3 & MJL & 24 & 14.5 & 12.9 \\
4 & CALB & 24 & 2.3 & 9.4 \\
5 & MML & 24 & 9.8 & 9.6 \\
\hline
\end{tabular}

2011 2012 年, 何延红和官智课题组 ${ }^{[13 ~ 15]}$ 分别报 道了使用地衣芽孢杆菌碱性蛋白酶(BLAP)、宇佐美曲䨋 酸性蛋白酶和木瓜凝乳蛋白酶(Chymopapain)在有机溶 剂和水的混合介质中可以催化芳香醛 19 和环酮 18 的不 对称 aldol 反应(Eq. 7), 产物的收率在 $28 \% \sim 92 \%, e e$ 值 达到 $22 \% \sim 99 \%$. 之后该课题组在研究杂环酮和芳香醛 的不对称 aldol 反应时, 发现猪胰脂肪酶 II (PPL II), 小 麦胚芽脂肪酶, 黑曲霉天野脂肪酶 A, 猪胰蛋白酶在水 和乙腈的混合介质中 $30{ }^{\circ} \mathrm{C}$ 下均能催化该反应 ${ }^{[29]}$, 收率 范围为 31\% 59\%, ee 值 45\% 87\%.

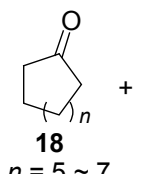<smiles>[R]C=O</smiles>
$\stackrel{\text { Hydrolase }}{\longrightarrow}$ $n=5 \sim 7$<smiles>[R]C(O)C1CCCC1=O</smiles>

虽然酶非专一性催化 aldol 反应已有大量报道, 但 是酶非专一性催化 aldol 缩合反应的报道却很少, 林贤 福等 ${ }^{[30]}$ 以咪唑为助催化剂, 使用 $D$-酰化氨基酸水解酶 在异辛烷或者辛烷为溶剂催化芳香醛 19 和丙酮 16 的差 醛缩合反应(Eq. 8), 产物以 $E$ 构型为主, 反应的收率最 高接近 $100 \%$.

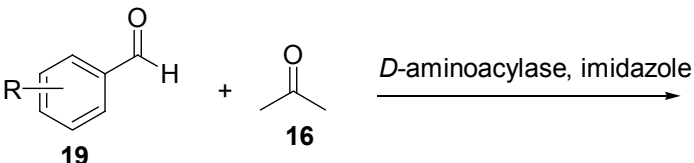<smiles>[R][X]1ccc(C=CC(C)=O)cc1</smiles>

21

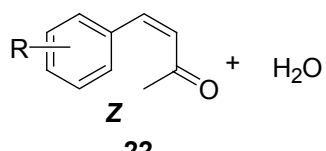

22
$\mathrm{R}=p-\mathrm{NO}_{2}, 67.8 \%, E / Z=38 / 1 ; \quad \mathrm{R}=m-\mathrm{NO}_{2}, 88.8 \%, E / Z=224 / 1 ;$

$\mathrm{R}=o-\mathrm{NO}_{2}, 44.4 \%, E / Z=1 / 1 ; \quad \mathrm{R}=\mathrm{H}, 74.3 \%, E / Z=245 / 1 ;$

$\mathrm{R}=p-\mathrm{Cl}, 90 \%, E ; \quad \mathrm{R}=m-\mathrm{Cl}, 99.6 \%, E / Z=57 / 1$

$\mathrm{R}=o-\mathrm{Cl}, 39 \%, E / Z=282 / 1 ; \quad \mathrm{R}=p-\mathrm{OH}, 8.5 \%, E / \mathrm{Z}=90 / 1$;

$\mathrm{R}=m-\mathrm{OH}, 0.6 \%, E ; \quad \mathrm{R}=0-\mathrm{OH}, 20.4 \%, E$;

$\mathrm{R}=p-\mathrm{OCH}_{3}, 89.8 \%, E / Z=19 / 1 \mathrm{R}=p-\mathrm{CH}_{3}, 87.1 \%, E / Z=12 / 1$

\subsubsection{Knoevenagel 反应}

Knoevenagel 反应是由碱催化的缩合反应，反应机 理非常类似 aldol 缩合反应. 2010 年我们课题组 ${ }^{[16]}$ 在尝 试使用水解酶催化芳香醛 19 与氰基乙酸甲酯 23 的 Knoevenagel 反应时, 发现在醇类溶剂中猪胰脂肪酶 (PPL)不仅催化 Knovenagel 反应，还催化酯交换反应， 反应的收率接近 100\%(Eq. 9). 通过对反应的动力学研 究发现 Knoevenagel 缩合反应速率明显快于酯交换的反 应速率，因此可以证明：在有机溶剂中，脂肪酶非专一 性催化 Knoevenagel 的能力要远强于其天然属性一催化 酯化的能力. 在 2011 年何延红和官智等 ${ }^{[31]}$ 将酶的非专 一性催化 Knoevenagel 反应和酯交换的串联反应应用于 2H-1-苯并吡喃-2-酮类化合物制备. $2 H$-1-苯并吡喃-2-酮 类化合物具有良好生理活性和药理活性, 是杀鼠灵的重 要中间体，也具有抗癌等作用. 他们使用地衣芽孢杆菌 碱性蛋白酶(BLAP)催化水杨醛 25 和乙酰乙酸乙酯 26 的 Knoevenagel 和分子内酯交换反应制备 $2 H-1$-苯并吡 喃-2-酮类衍生物 28(表 4). 在 DMSO 和水 $(V: V=9: 1$, 的混合溶剂中，水杨醛 25 和乙酰乙酸乙酯 26 的物质的 量比为 $1: 3$, 反应温度 $55{ }^{\circ} \mathrm{C}$, 主产物的收率在 $10 \%$ $75 \%$ 之间.

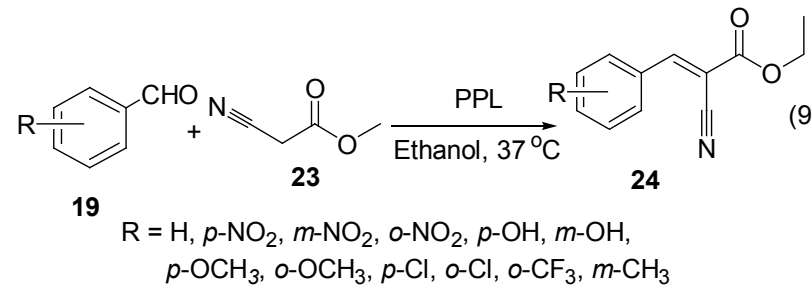

2012 年何延红等 ${ }^{[17]}$ 用木瓜蛋白酶在 DMSO 和水混 合介质中催化芳香醛、 $\alpha, \beta$-不饱和芳香醛与乙酰乙酸乙 酯、乙酰丙酮的 Knovenegal 反应，产物的收率最高可到 达 $87 \%, Z / E$ 选择性最高可到达 100：0. 该课题组 ${ }^{[32]}$ 还 
表 4 BLAP 催化 Knoevenagel 和分子内酯交换串联反应

Table 4 BLAP-catalyzed domino Knoevenagel/intramolecular transesterification reaction
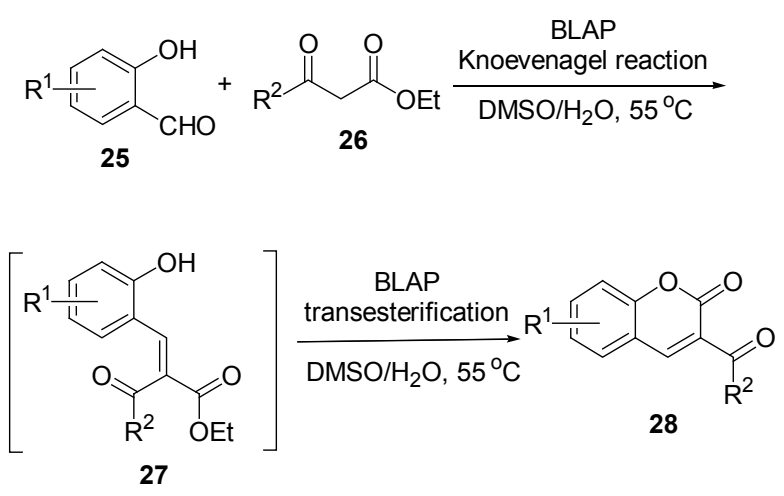

\begin{tabular}{|c|c|c|c|}
\hline Entry & 25 & $\mathrm{R}^{2}$ & Yield/\% \\
\hline $\begin{array}{l}1 \\
2 \\
3\end{array}$ & & $\begin{array}{c}\mathrm{Ph} \\
\mathrm{OEt} \\
\mathrm{CH}_{3}\end{array}$ & $\begin{array}{l}75 \\
68 \\
60\end{array}$ \\
\hline $\begin{array}{l}4 \\
5 \\
6\end{array}$ & & $\begin{array}{c}\mathrm{Ph} \\
\mathrm{OEt} \\
\mathrm{CH}_{3}\end{array}$ & $\begin{array}{l}73 \\
69 \\
58\end{array}$ \\
\hline $\begin{array}{l}7 \\
8 \\
9\end{array}$ & & $\begin{array}{c}\mathrm{Ph} \\
\mathrm{OEt} \\
\mathrm{CH}_{3}\end{array}$ & $\begin{array}{l}45 \\
56 \\
30\end{array}$ \\
\hline $\begin{array}{l}10 \\
11\end{array}$ & & $\begin{array}{c}\mathrm{Ph} \\
\mathrm{OEt}\end{array}$ & $\begin{array}{l}48 \\
25\end{array}$ \\
\hline 12 & & $\mathrm{Ph}$ & 18 \\
\hline 13 & & $\mathrm{OEt}$ & 10 \\
\hline 14 & & $\mathrm{CH}_{3}$ & 48 \\
\hline
\end{tabular}

报道了地衣芽狍杆菌碱性蛋白酶(BLAP)催化杂环芳香 醛与乙酰乙酸乙酯、乙酰丙酮的 Knovenegal 反应, 发现 具有较好的底物适应性和较高的收率.

\section{1 .4 其他单元反应}

在有机合成中, Henry 反应是一个非常有效地构造 $\mathrm{C}-\mathrm{C}$ 键的反应. 2010 年林贤福等 ${ }^{[19]}$ 发现 $D$-酰化氨基酸 水解酶可以在 DMSO 溶剂中催化烷烃硝基 29 和芳香醛 15 的 Henry 反应(Eq. 10), 收率范围为 $15 \% \sim 99 \%$, 但是
该反应没有对映选择性. 何延红等 ${ }^{[18]}$ 也报道了水解酶 可以催化 Henry 反应，也收率达到 $64 \%$, 没有对映选择 性.

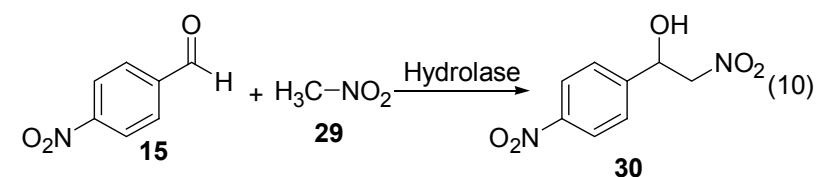

Condition A: $D$-aminoacylase, DMSO, $50{ }^{\circ} \mathrm{C}$, yield $99 \%$ Condition B: Immobilized lipase from Thermomyces lanuginosus $10 \%$ water and $90 \%$ cyclohexane, r.t., yield $64 \%$

aza-Diels-Alder 反应是一个构建含氮六元环的重要 反应, 含氮六元环在药物和天然产物中有着重要的应 用，该反应主要是经历 Mannich-Michael 反应历程. 何 延红和官智等 ${ }^{[33]}$ 用卵清溶菌酶(HEWL)在乙腈和水的混 合介质中催化芳香醛 19、芳香胺 31 和环已-2-烯酮(32) 三组分的 aza-Diels-Alder 反应(Eq. 11), 反应的收率可达 98\%, endo/exo 选择性达到 $90 ： 10$.
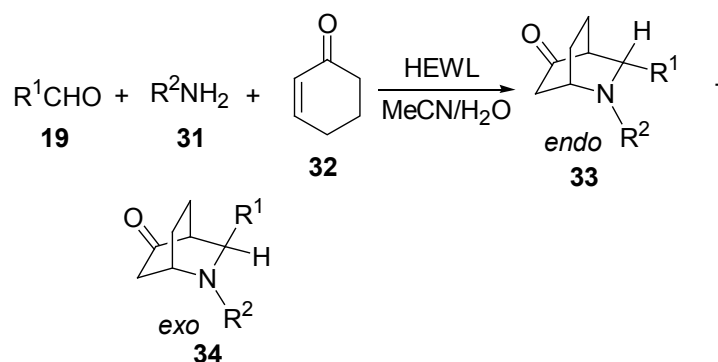

32

33

\section{2 两步反应}

\subsection{1 催化脱羧-aldol 反应/Knoevenagel 反应}

余孝其等 ${ }^{[34]}$ 报道了用 CALB 催化芳香醛 19 与 1,3二羰基化合物 35 脱羧的 aldol 反应和脱羒的 Knoevenagel 反应(表 5). 在该反应中加入了微量的 1,4,7,10-四氮杂环十二烷作为添加剂, 在乙腈溶液中 20 ${ }^{\circ} \mathrm{C}$ 反应 $20 \mathrm{~h}$ 得到脱羧的 aldol 反应产物 $\mathbf{3 6}$, 收率在 $81 \% \sim 96 \%$; 当反应体系为微量的水和 0.5 equiv. 的苯胺 时, 在乙腈溶液中 $30{ }^{\circ} \mathrm{C}$, 反应 $24 \mathrm{~h}$ 能得到脱羧的 Knoevenagel 加成产物 37, 收率为 $80 \%$ 左右. 从中可以 看出脂肪酶在不同的反应条件下可以具有不同的催化 
活性，可以通过条件的改变来控制酶的催化活性.

表 5 脂肪酶在不同条件下催化的脱羧的 aldol 反应和脱羧的 Knoevenagel 反应

Table 5 Lipase-catalyzed decarboxylative aldol reaction and decarboxylative Knoevenagel reaction

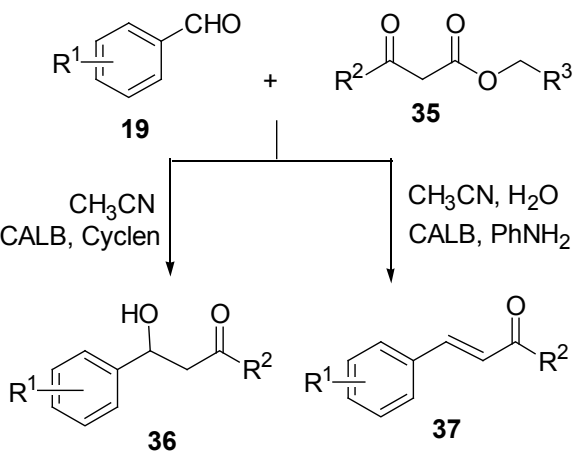

\begin{tabular}{|c|c|c|c|c|}
\hline \multirow{2}{*}{ Entry } & \multirow{2}{*}{$\mathrm{R}^{1}$} & \multirow{2}{*}{ Subtract 35} & \multicolumn{2}{|c|}{ Yield/\% } \\
\hline & & & 36 & 37 \\
\hline 1 & $p-\mathrm{NO}_{2}$ & & 96 & 90 \\
\hline 2 & $o-\mathrm{NO}_{2}$ & & 81 & 85 \\
\hline 3 & $m-\mathrm{NO}_{2}$ & & 87 & 88 \\
\hline 4 & $p-\mathrm{NO}_{2}$ & & 97 & 91 \\
\hline 5 & $p-\mathrm{NO}_{2}$ & & 95 & 88 \\
\hline 6 & $p-\mathrm{NO}_{2}$ & & 94 & 86 \\
\hline 7 & $p-\mathrm{NO}_{2}$ & & 91 & 82 \\
\hline 8 & $p-\mathrm{NO}_{2}$ & & - & - \\
\hline 9 & $p-\mathrm{NO}_{2}$ & & 95 & 85 \\
\hline 10 & $p-\mathrm{NO}_{2}$ & & 87 & 84 \\
\hline
\end{tabular}

\subsection{2 催化缩合一加成反应}

Berglund 等 ${ }^{[4 a]}$ 最早发现 CALB-Ser105Ala 可以催化 aldol 反应，反应的机理是：形成烯醇式中间体，与酶上 活性位点的氧负离子空洞和催化三联体作用进行反应. 但是该反应没有对映选择性，反应时间过长。之后
Berglund 等 ${ }^{[4 \mathrm{~b}]}$ 继续报道了用 CALB-Ser105Ala 催化 1,3羰基化合物与 $\alpha, \beta$-不饱和羰基化合物的 Michael 加成. 由于 CALB-Ser105Ala 催化 aldol 反应的活性很低, Berglund 等 ${ }^{[28]}$ 设计了一个串联反应改变 aldol 反应的平衡, 来观察 aldol 反应速率是否会提高. 丙醛 38 通过 CALB-Ser105Ala 催化发生 aldol 反应，然后用分子篎脱 水生成 $\alpha, \beta$-不饱和羰基化合物 $\mathbf{4 0}$, 继续和硫醇发生 Michael 加成, 结果发现不是平衡影响 aldol 反应速率 (Scheme 2).
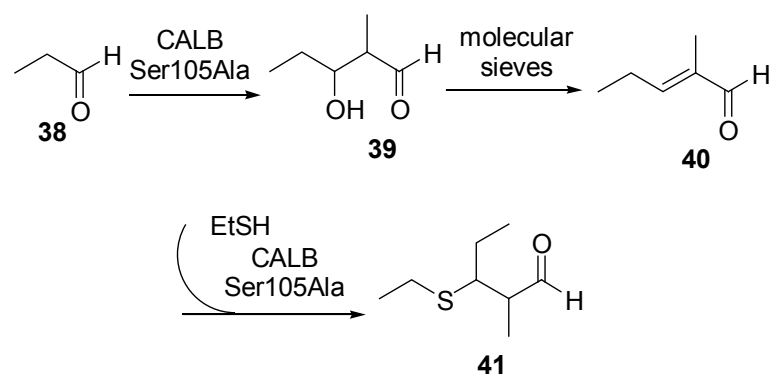

Scheme 2

Gupta 等 ${ }^{[35]}$ 报道了 Novozym 435 在吡啶溶剂中催化 乙酸乙烯酯(42)原位水解成乙醛 43 , 继而与三环二酮 44 发生反应最终生成产物 $47,4 \mathrm{~h}$ 后反应的收率 $97 \%$ (Scheme 3). 该反应的历程实际是经历了原位水解、非 专一性催化缩合反应和加成两步串联反应.
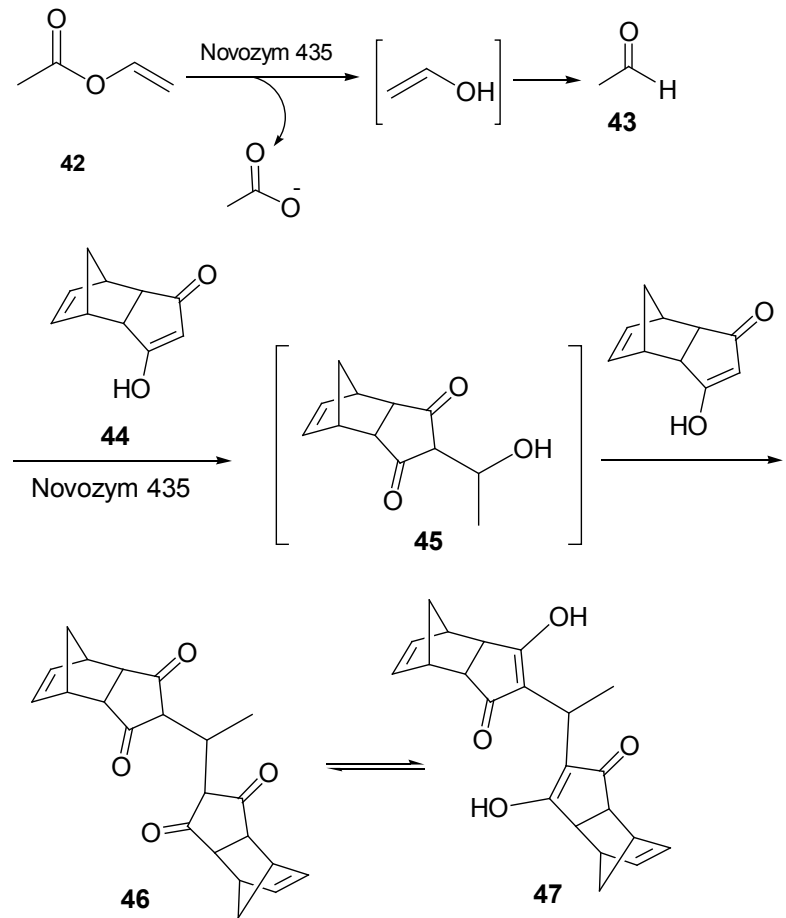

Scheme 3 


\subsection{3 串联 Michael 加成一酰化反应}

1986 年 Kitazume 等 ${ }^{[6]}$ 在 $\mathrm{pH}=8.0$ 的缓冲溶液中, 用 猪肝酯酶和柱状假丝酵母脂肪酶成功催化合成具有手 性 Michael 加成的产物的时候, 发现当以 2-三氟甲基丙 烯酸(3)和含有胺基与亲核取代基团的芳香化合物 48 原 料, 还催化合成具有手性中心杂环化合物 49, 该反应主 要是通过手性的 Michael 加成和酰化反应完成(表 6).

林贤福等 ${ }^{[36]}$ 发现枯草芽孢杆菌碱性蛋白酶在无水 吡啶中 $50{ }^{\circ} \mathrm{C}$ 下可以催化乙烯基-3-丙烯酸基丙酸酯(50), $D$-葡萄糖(51)和咪唑衍生物 53 反应生成一系列咪唑含 糖衍生物 25 (Scheme 4), 该反应先经历了酰化反应生成 6- $O$-( $\beta$-丙烯酸基-丙酰基)- $D$-葡萄糖(52), 然后再和咪唑 衍生物发生 Michael 加成得到产物 54, 收率在 $69 \%$ $80 \%$.

\subsection{4 串联 Michael 加成一环化反应}

余孝其等 ${ }^{[37]}$ 发现了利用单一脂肪酶的非专一性催 化一锅化法可以合成二氢呋喃衍生物, 用猪胰脂肪酶 (PPL)在 DMSO 和水 $(V: V=4: 1)$ 的混合溶剂中催化硝 基苯乙烯 55 和乙酰丙酮 56, 发现除了发生 Michael 加 成, 还发生环化反应得到二氢呋喃的衍生物 $\mathbf{5 7}$, 反应的 收率为 $50 \% \sim 85 \%$, 产物以 $Z$ 构型为主, $Z / E$ 选择性最高 大于 $99: 1$ (表 7).

\section{3 一锅三组分反应}

吲哚啉螺环化合物是由吲哚啉和哌啶组成的螺环 化合物, 由于其具有非常重要的潜在生理活性和药理活 性, 常用于农药和医药领域. 章鹏飞等 ${ }^{[38]}$ 研究了猪胰脂

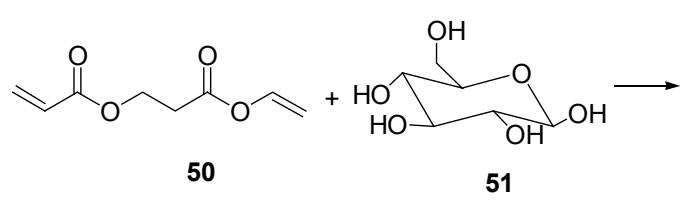<smiles>C=CC(=O)OCCC(=O)OCC(O)C(O)C(O)OC(=O)CCCCC</smiles><smiles>N#CCC(=O)OCCC(=O)OC1C(O)C(O)C(O)C(O)C1O</smiles>

54<smiles></smiles>

\section{Scheme 4}

肪酶(PPL)在乙醇溶剂中催化吲哚酮 58、腈基化合物 59 和 1,3-二羰基化合物 60 一锅法合成吲哚啉螺环衍生物 61(表 8), 该反应先后经历了 Knoevengel 反应、Michael 加成和环合反应，在水含量 $10 \% \sim 20 \%$ 时脂肪酶的催化 活性最高，反应的收率在 $83 \% \sim 95 \%$.

基于相同的反应历程，该课题组 ${ }^{[39]}$ 还报道了猪胰 脂肪酶(PPL)在乙醇中催化芳醛 19、腈基化合物 59 及 1,3-二羰基化合物 60 为底物的三组分一锅法合成四氢 苯并吡喃衍生物 62 的研究(Eq. 12), 反应的收率最高达 到 $97 \%$. 苯并吡喃类化合物具有良好生理活性和药理活 性, 如解痉、利尿、抗凝血剂、抗癌等作用.

表 6 水解酶催化的 Michael 加成反应

Table 6 Michael addition catalyzed by hydrolase
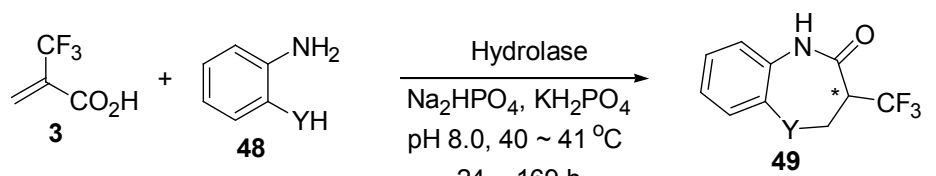

$24 \sim 169 \mathrm{~h}$

\begin{tabular}{|c|c|c|c|c|}
\hline Subtrate & Enzyme & Time $/ \mathrm{h}$ & Yield/\% & $e e / \%$ \\
\hline \multirow{3}{*}{ 2-Aminophenol } & Candida cylindracea Lipase & 21 & 83 & 41 \\
\hline & Pig liver esterase & 16 & 69 & 36 \\
\hline & Trichoderma viride Lipase & 2.5 & 94 & 56 \\
\hline \multirow{2}{*}{ 2-Aminothiophenol } & Candida cylindracea Lipase & 20 & 86 & $47(S)$ \\
\hline & Trichoderma viride Lipase & 6 & 72 & $36(S)$ \\
\hline \multirow[b]{2}{*}{$o$-Phenylenediamine } & Candida cylindracea Lipase & 24 & 56 & 38 \\
\hline & Trichoderma viride Lipase & 24 & 52 & 44 \\
\hline \multirow{2}{*}{ 4-Methyl-2-aminophenol } & Candida cylindracea Lipase & 20 & 71 & 25 \\
\hline & Trichoderma viride Lipase & 20 & 57 & 37 \\
\hline
\end{tabular}


表 7 脂肪酶催化硝基苯乙烯和乙酰丙酮的反应

Table 7 Lipase-catalyzed reaction of nitrostyrene and acetylacetone

\begin{tabular}{|c|c|c|c|c|c|}
\hline \multirow{2}{*}{ Entry } & \multirow{2}{*}{$\mathrm{R}^{1}$} & \multicolumn{2}{|c|}{$n=0$} & \multicolumn{2}{|r|}{$n=1$} \\
\hline & & Yield/\% & $Z / E$ & Yield/\% & $Z / E$ \\
\hline 1 & $\mathrm{C}_{6} \mathrm{H}_{5}$ & 61.7 & $>99: 1$ & 64.3 & $>99: 1.0$ \\
\hline 2 & $4-\mathrm{CH}_{3} \mathrm{C}_{6} \mathrm{H}_{4}$ & 51.4 & $>99: 1$ & 54.6 & $71: 29$ \\
\hline 3 & $4-\mathrm{CH}_{3} \mathrm{OC}_{6} \mathrm{H}_{4}$ & 63.6 & $>99: 1$ & 73.8 & $>99: 1.0$ \\
\hline 4 & $4-\mathrm{FC}_{6} \mathrm{H}_{4}$ & 48.9 & $>99: 1$ & 63.5 & $91: 9.0$ \\
\hline 5 & $4-\mathrm{ClC}_{6} \mathrm{H}_{4}$ & 50.6 & $>99: 1$ & 70.8 & $65: 35$ \\
\hline 6 & 4- $\mathrm{BrC}_{6} \mathrm{H}_{4}$ & 50.7 & $>99: 1$ & 61.2 & $64: 36$ \\
\hline 7 & $4-\mathrm{CF}_{3} \mathrm{C}_{6} \mathrm{H}_{4}$ & 52.3 & $96: 4$ & 65.4 & $46: 54$ \\
\hline 8 & $2-\mathrm{ClC}_{6} \mathrm{H}_{4}$ & 80.6 & $>99: 1$ & 85.7 & $28: 72$ \\
\hline 9 & $3-\mathrm{ClC}_{6} \mathrm{H}_{4}$ & 54.5 & $97: 3$ & 65.6 & $>99: 1.0$ \\
\hline
\end{tabular}

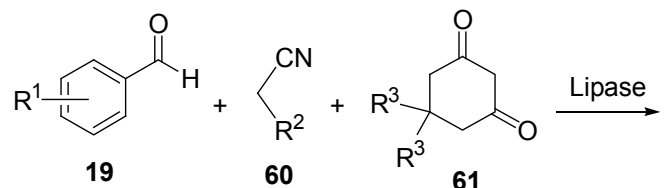<smiles>[R]C1=C(N)OC2=C(C(=O)CC([R])([R])C2)C1c1ccc[R1]([H])c1</smiles>

Mannich 反应是形成 $\mathrm{C}-\mathrm{C}$ 键和 $\mathrm{C}-\mathrm{N}$ 键的重要反 应，通过该反应合成一系列氨基酸、氨基醇及其衍生物 被广泛应用于许多复杂的天然或者药物中间体的合成 中. 2009 年余孝其等 ${ }^{[40]}$ 发现一些脂肪酶可以催化芳香醛 19、芳香胺 31、丙酮 14 的三组分 Mannich 反应，其中 催化活性最好的为米赫毛霉脂肪酶(MML), 反应的收率 最高达到 $89.1 \%$. 该反应在有水的环境中能够增加酶的 催化多功能活性, 在无水的情况下酶几乎不显示出 Mannich 反应催化活性. 2010 年何延红和官智等 ${ }^{[41]}$ 也发 现脂肪酶可以催化芳香醛、芳香胺、环己酮的 Mannich 反应，发现在 $30{ }^{\circ} \mathrm{C}$ 下, 在乙醇和水的混合介质中, 假 丝酵母脂肪酶(CRL)的催化活性最高, 收率最高可以达 到 94\%. 章鹏飞等 ${ }^{[42]}$ 尝试用胰蛋白酶(Trypsin)在丙酮溶 剂中催化 Mannich 反应, 该反应具有很好的收率和底物 适应性，同时发现在无水条件下胰蛋白酶催化 Mannich 反应活性最高，说明水不是该反应所必须的(表 9).

不对称 Mannich 反应主要用于制备手性 $\beta$-氨基酮类 和醛类. 2012 年何延红和官智等 ${ }^{[43]}$ 首先发现了灰色链霉 菌蛋白酶 XIV (SGP)在乙腈和水的混合介质中 $30{ }^{\circ} \mathrm{C}$ 下
表 8 脂肪酶催化一锅法合成四氢苯并吡喃衍生物

Table 8 One-pot synthesis of tetrahydrochromene derivatives catalyzed by lipase
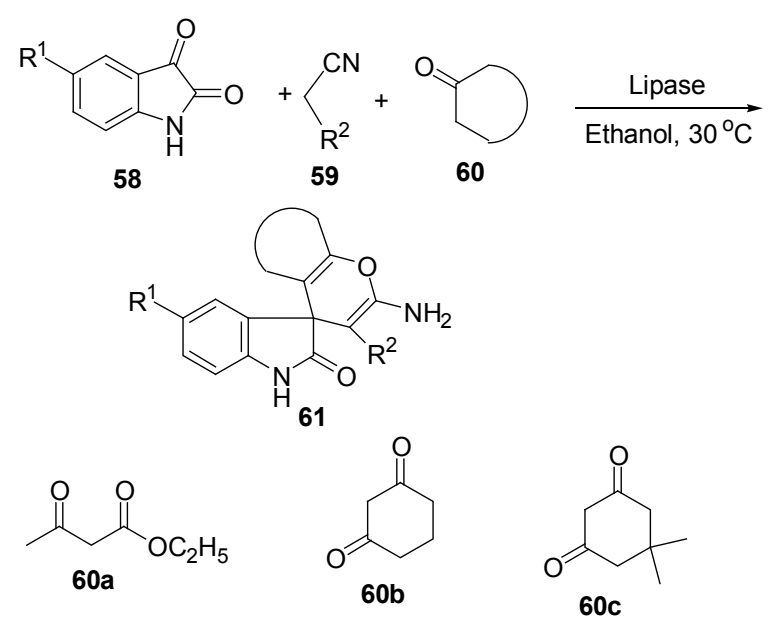

\begin{tabular}{cllcc}
\hline Entry & $\mathrm{R}^{1}$ & \multicolumn{1}{c}{$\mathrm{R}^{2}$} & Compd. 60 & Yield $/ \%$ \\
\hline 1 & $\mathrm{H}$ & $\mathrm{CN}$ & $\mathbf{6 0 a}$ & 92 \\
2 & $\mathrm{H}$ & $\mathrm{CN}$ & $\mathbf{6 0 b}$ & 93 \\
3 & $\mathrm{H}$ & $\mathrm{CN}$ & $\mathbf{6 0 c}$ & 94 \\
4 & $\mathrm{H}$ & $\mathrm{COOEt}$ & $\mathbf{6 0 a}$ & 87 \\
5 & $\mathrm{H}$ & $\mathrm{COOEt}$ & $\mathbf{6 0 b}$ & 85 \\
6 & $\mathrm{H}$ & $\mathrm{COOEt}$ & $\mathbf{6 0 c}$ & 88 \\
7 & $\mathrm{Cl}$ & $\mathrm{CN}$ & $\mathbf{6 0 a}$ & 93 \\
8 & $\mathrm{Cl}$ & $\mathrm{CN}$ & $\mathbf{6 0 b}$ & 95 \\
9 & $\mathrm{Cl}$ & $\mathrm{CN}$ & $\mathbf{6 0 c}$ & 95 \\
10 & $\mathrm{Cl}$ & $\mathrm{COOEt}$ & $\mathbf{6 0 a}$ & 85 \\
11 & $\mathrm{Cl}$ & $\mathrm{COOEt}$ & $\mathbf{6 0 b}$ & 83 \\
12 & $\mathrm{Cl}$ & $\mathrm{COOEt}$ & $\mathbf{6 0 c}$ & 86 \\
13 & $\mathrm{OMe}$ & $\mathrm{CN}$ & $\mathbf{6 0 a}$ & 93 \\
14 & $\mathrm{OMe}$ & $\mathrm{CN}$ & $\mathbf{6 0 b}$ & 95 \\
15 & $\mathrm{OMe}$ & $\mathrm{CN}$ & $\mathbf{6 0 c}$ & 93 \\
16 & $\mathrm{OMe}$ & $\mathrm{COOEt}$ & $\mathbf{6 0 b}$ & 88 \\
17 & $\mathrm{OMe}^{2}$ & $\mathrm{COOEt}$ & $\mathbf{6 0 c}$ & 82 \\
18 & $\mathrm{NO}_{2}$ & $\mathrm{CN}$ & $\mathbf{6 0 a}$ & 91 \\
19 & $\mathrm{NO}_{2}$ & $\mathrm{CN}$ & $\mathbf{6 0 b}$ & 94 \\
20 & $\mathrm{NO}_{2}$ & $\mathrm{COOEt}$ & $\mathbf{6 0 a}$ & 95 \\
\hline
\end{tabular}

可以催化芳香醛 19、芳香胺 31 和环酮 64 的不对称 Mannich 反应(Eq. 13). 收率最高可以达到 $92 \%$, ee 达到 $88 \%$. 反应中用尿素、 $\mathrm{Cu}^{2+}$ 和 $\mathrm{Ag}^{2+}$ 让 SGP 失活, 发现不 影响反应的收率，但是几乎没有立体选择性，这暗示影 响 Mannich 反应的立体选择性主要是 SGP 的天然的空 间折叠结构.

1,4-二氢吡啶一般都是用醋酸铵或者液氨通过 Hantzsch 反应获得, 2012 年林贤福等 ${ }^{[44]}$ 用 CALB 催化乙 酰胺 67、醛类 4 和 1,3-二羰基化合物 66 三组分化合物, 在 $50{ }^{\circ} \mathrm{C}$ 下以甲基叔丁基醚为溶剂, 生成 1,4-二氢吡啶 衍生物 68, 收率范围为 17\% 93\% (Eq. 13). 1,4-二氢吡 啶衍生物是吡啶类药物的重要中间体，如硝苯地平，在 该反应中乙酰胺首次被当做胺源制备 $1,4-$ 二氢吡啶衍生 
表 9 酶催化的 Mannich 反应

Table 9 Enzyme-catalyzed Mannich reaction

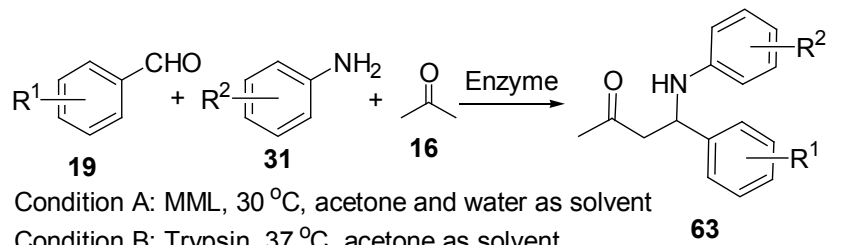
Condition B: Trypsin, $37^{\circ} \mathrm{C}$, acetone as solvent

\begin{tabular}{clllc}
\hline \multirow{2}{*}{ Entry } & \multirow{2}{*}{$\mathrm{R}^{1}$} & \multirow{2}{*}{$\mathrm{R}^{2}$} & \multicolumn{2}{c}{ Yield/\% } \\
\cline { 4 - 5 } & $\mathrm{H}$ & $\mathrm{H}$ & 87.3 & - \\
\hline 1 & $m-\mathrm{NO}_{2}$ & $\mathrm{H}$ & 82.4 & - \\
2 & $p-\mathrm{NO}_{2}$ & $\mathrm{H}$ & 83.5 & - \\
3 & $p-\mathrm{OCH} \mathrm{B}_{3}$ & $\mathrm{H}$ & 89.1 & - \\
4 & $p-\mathrm{OH}$ & $\mathrm{H}$ & 43.6 & - \\
5 & $p-\mathrm{CN}$ & $\mathrm{H}$ & 65.3 & - \\
6 & $p-\mathrm{Cl}$ & $\mathrm{H}$ & 83.1 & - \\
7 & $\mathrm{H}$ & $o-\mathrm{OCH}_{3}$ & - & 68 \\
8 & $\mathrm{H}$ & $p-\mathrm{Cl}^{[42]}$ & - & 74 \\
9 & $\mathrm{H}$ & $m-\mathrm{Cl}_{1}$ & - & 65 \\
10 & $\mathrm{H}$ & $m-\mathrm{NO}_{2}$ & - & 47 \\
11 & $p-\mathrm{NO}_{2}$ & $p-\mathrm{OCH}_{3}$ & - & 93 \\
12 & $p-\mathrm{CH}_{3}$ & $p-\mathrm{OCH}_{3}$ & - & 45 \\
13 & $p-\mathrm{Cl}$ & $p-\mathrm{OCH}_{3}$ & - & 83 \\
14 & & & &
\end{tabular}

物.

\section{3 结语}

近十年, 酶非专一性催化研究的兴起, 拓展了生物 催化剂在有机合成中的应用, 为进一步研究酶的结构与 功能的关系提供了新的思路, 同时深入探讨了酶催化非 专一性的机理, 便于开发酶的新功能.随着研究的深入, 水解酶的非专一性催化在不对称合成和串联反应中不 断取得突破. 利用其非专一性催化功能, 可以串联两步 甚至多步反应，得到相对复杂的化合物结构，这一点， 是普通催化剂所难以比拟的.然而, 酶的非专一性催化 离产业化还有一定距离, 主要问题是, 缺乏系统的理论 指导, 并且水解酶酶催化可变条件太多, 催化效果不稳 定. 因此需要长期系统的研究, 把酶的非专一性催化模 式固定化，调控好溶剂、时间、含水量等各种因素另外, 酶的来源及制备成本对酶非专一性催化产业化也是至 关重要的考虑因素. 目前, 对酶的非专一性催化研究主 要集中在水解酶中的脂肪酶和蛋白酶, 如 PPL, CALB 和枯草杆菌碱性蛋白酶等, 这些酶相对便宜易得, 研究 它们的非专一性催化对于产业化更有应用价值.

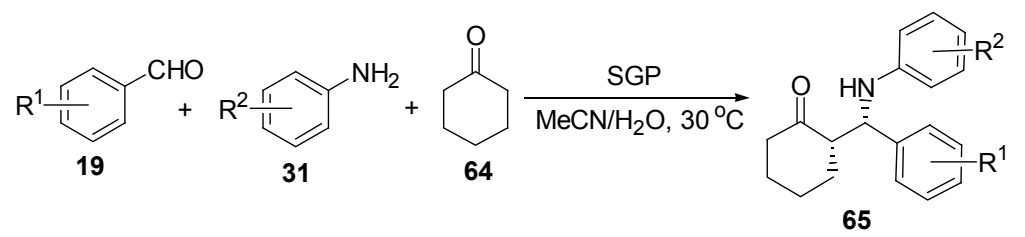

\footnotetext{
$\mathrm{R}^{1}=4-\mathrm{NO}_{2}, \mathrm{R}^{2}=\mathrm{H}, 96 \mathrm{~h}, 64 \%, d r($ syn:anti) $=88: 12$, ee(syn) 83\%; $\mathrm{R}^{1}=4-\mathrm{CF}_{3}, \mathrm{R}^{2}=\mathrm{H}, 94 \mathrm{~h}, 73 \%$, dr(syn:anti) = 81:19, ee(syn) $78 \%$; $R^{1}=4-B r, R^{2}=H, 94$ h, 65\%, dr(syn:anti) = 74:126, ee(syn) $76 \%$; $\mathrm{R}^{1}=4-\mathrm{Cl}, \mathrm{R}^{2}=\mathrm{H}, 120 \mathrm{~h}, 92 \%$, dr(syn:anti) $=78: 22$, ee(syn) $75 \%$; $\mathrm{R}^{1}=3-\mathrm{F}, \mathrm{R}^{2}=\mathrm{H}, 94 \mathrm{~h}, 72 \%, d r($ syn:anti) $=70: 30$, ee(syn) $74 \%$; $\mathrm{R}^{1}=4-\mathrm{F}, \mathrm{R}^{2}=\mathrm{H}, 94 \mathrm{~h}, 66 \%$, dr(syn:anti) $=66: 34$, ee(syn) $64 \%$; $\mathrm{R}^{1}=4-\mathrm{CN}, \mathrm{R}^{2}=\mathrm{H}, 120 \mathrm{~h}, 61 \%, d r($ syn:anti $)=58: 42$, ee $($ syn $) 68 \%$; $\mathrm{R}^{1}=\mathrm{H}, \mathrm{R}^{2}=\mathrm{H}, 100 \mathrm{~h}, 62 \%, \operatorname{dr}($ syn:anti) $=60: 40$, ee(syn) $61 \%$; $\mathrm{R}^{1}=4-\mathrm{CH}_{3}, \mathrm{R}^{2}=\mathrm{H}, 120 \mathrm{~h}, 68 \%, d r($ syn:anti) $=40: 60$, ee (syn) 33\%; $\mathrm{R}^{1}=4-\mathrm{NO}_{2}, \mathrm{R}^{2}=3-\mathrm{Br}, 144 \mathrm{~h}, 24 \%, d r($ syn:ant $i)=92: 8$, ee(syn) $88 \%$; $\mathrm{R}^{1}=4-\mathrm{NO}_{2}, \mathrm{R}^{2}=3-\mathrm{CH}_{3}, 123 \mathrm{~h}, 54 \%, d r$ (syn:anti) $=91: 9$, ee(syn) $83 \%$; $\mathrm{R}^{1}=4-\mathrm{NO}_{2}, \mathrm{R}^{2}=4-\mathrm{Cl}, 144 \mathrm{~h}, 47 \%, d r($ syn:ant $)=89: 11$, ee $($ syn) $83 \%$; $\mathrm{R}^{1}=4-\mathrm{NO}_{2}, \mathrm{R}^{2}=4-\mathrm{CH}_{3}, 120 \mathrm{~h}, 81 \%, \operatorname{dr}$ (syn:anti) $=90: 10$, ee(syn) $82 \%$; $\mathrm{R}^{1}=4-\mathrm{NO}_{2}, \mathrm{R}^{2}=4-\mathrm{OCH}_{3}, 117 \mathrm{~h}, 71 \%$, dr(syn:ant $\left.i\right)=72: 28$, ee(syn) $72 \%$; $\mathrm{R}^{1}=4-\mathrm{Br}, \mathrm{R}^{2}=4-\mathrm{OCH}_{3}, 165 \mathrm{~h}, 66 \%, d r($ syn:anti $)=52: 48$, ee $($ syn $) 40 \%$
}

\section{References}

[1] (a) Copley, S. D. Curr. Opin. Chem. Biol. 2003, 7(2), 265.

(b) Kazlauskas, R. J. Curr. Opin. Chem. Biol. 2005, 9(2), 195.

(c) Khersonsky, O.; Roodveldt, C.; Tawfik, D. S. Curr. Opin. Chem. Biol. 2006, 10(5), 498.

(d) Hult, K.; Berglund, P. Trends Biotechnol. 2007, 25(5), 231.

(e) Xu, J. M.; Lin, X. F. Chin. J. Org. Chem. 2007, 27(12), 1473 (in Chinese)
(许建明, 林贤福, 有机化学, 2007, 27(12), 1473.)

(f) Babtie, A.; Tokuriki, N.; Hollfelder, F. Curr. Opin. Chem. Biol. 2010, 14(2), 200.

[2] Nam, H.; Lewis, N. E.; Lerman, J. A.; Lee, D. H.; Chang, R. L.; Kim, D.; Palsson, B. O. Science 2012, 337(6098), 1101.

[3] Carlqvist, P.; Svedendahl, M.; Branneby, C.; Hult, K.; Brinck, T.; Berglund, P. ChemBioChem 2005, 6(2), 331.

[4] (a) Branneby, C.; Carlqvist, P.; Magnusson, A.; Hult, K.; Brinck, T.; Berglund, P. J. Am. Chem. Soc. 2003, 125(4), 874. 
(b) Svedendahl, M.; Hult, K.; Berglund, P. J. Am. Chem. Soc. 2005, 127(51), 17988

[5] Svedendahl, M.; Jovanovic, B.; Fransson, L.; Berglund, P. Chem CatChem 2009, 1(2), 252.

[6] Kitazume, T.; Ikeya, T.; Murata, K. J. Chem. Soc., Chem. Commun. 1986, (17), 1331.

[7] Cai, Y.; Yao, S. P.; Wu, Q.; Lin, X. F. Biotechnol. Lett. 2004, 26(6), 525.

[8] de Souza, R.; Matos, L. M. C.; Goncalves, K. M.; Costa, I. C. R.; Babics, I.; Leite, S. G. F.; Oestreicher, E. G.; Antunes, O. A. C. Tetrahedron Lett. 2009, 50(17), 2017.

[9] Xu, J. M.; Zhang, F.; Wu, Q.; Zhang, Q. Y.; Lin, X. F. J. Mol. Catal., B: Enzym. 2007, 49(1 4), 50.

[10] Strohmeier, G. A.; Sovic, T.; Steinkellner, G.; Hartner, F. S.; Andryushkova, A.; Purkarthofer, T.; Glieder, A.; Gruber, K.; Griengl, H. Tetrahedron 2009, 65(29 30), 5663.

[11] Li, C.; Feng, X. W.; Wang, N.; Zhou, Y. J.; Yu, X. Q. Green Chem. 2008, 10(6), 616.

[12] Li, C.; Zhou, Y. J.; Wang, N.; Feng, X. W.; Li, K.; Yu, X. Q. J. Biotechnol. 2010, 150(4), 539.

[13] Li, H. H.; He, Y. H.; Guan, Z. Catal. Commun. 2011, 12(7), 580.

[14] Xie, B. H.; Li, W.; Liu, Y.; Li, H. H.; Guan, Z.; He, Y. H. Tetrahedron 2012, 68(15), 3160.

[15] He, Y. H.; Li, H. H.; Chen, Y. L.; Xue, Y.; Yuan, Y.; Guan, Z. Adv. Synth. Catal. 2012, 354(4), 712.

[16] Lai, Y. F.; Zheng, H.; Chai, S. J.; Zhang, P. F.; Chen, X. Z. Green Chem. 2010, 12(11), 1917.

[17] Hu, W.; Guan, Z.; Deng, X.; He, Y. H. Biochimie 2012, 94(3), 656.

[18] Tang, R. C.; Guan, Z.; He, Y. H.; Zhu, W. J. Mol. Catal, B: Enzym. 2010, 63(1 2), 62 .

[19] Wang, J. L.; Li, X.; Xie, H. Y.; Liu, B. K.; Lin, X. F. J. Biotechnol. 2010, 145(3), 240

[20] Zaks, A.; Klibanov, A. M. Science 1984, 224(4654), 1249.

[21] Zaks, A.; Klibanov, A. M. J. Biol. Chem. 1988, 263(17), 8017.

[22] Cai, Y.; Wu, Q.; Xiao, Y. M.; Lv, D. S.; Lin, X. F. J. Biotechnol. 2006, $121(3), 330$.

[23] Xu, J. M.; Zhang, F.; Liu, B. K.; Wu, Q.; Lin, X. F. Chem. Commun. 2007, (20), 2078.

[24] Wang, J. L.; Xu, J. M.; Wu, Q.; Lv, D. S.; Lin, X. F. Tetrahedron
2009, 65(12), 2531.

[25] (a) Wu, W. B.; Xu, J. M.; Wu, Q.; Lv, D. S.; Lin, X. F. Synlett 2005, (16), 2433

(b) Wu, W. B.; Xu, J. M.; Wu, Q.; Lv, D. S.; Lin, X. F. Adv. Synth. Catal. 2006, 348(4 5), 4872.

[26] Torre, O.; Alfonso, I.; Gotor, V. Chem. Commun. 2004, (15), 1724.

[27] Madalinska, L.; Kwiatkowska, M.; Cierpial, T.; Kielbasinski, P. J. Mol. Catal., B: Enzym. 2012, 81, 25.

[28] Branneby, C.; Carlqvist, P.; Hult, K.; Brinck, T.; Berglund, P., J. Mol. Catal., B: Enzym. 2004, 31(4 6), 123.

[29] Guan, Z.; Fu, J. P.; He, Y. H. Tetrahedron Lett. 2012, 53 (37), 4959.

[30] Chen, X. A.; Liu, B. K.; Kang, H.; Lin, X. F. J. Mol. Catal., B: Enzym. 2011, 68(1), 71.

[31] Wang, C. H.; Guan, Z.; He, Y. H. Green Chem. 2011, 13(8), 2048.

[32] Xie, B. H.; Guan, Z.; He, Y. H. Biocatal. Biotransfor 2012, 30(2), 238.

[33] He, Y. H.; Hu, W.; Guan, Z. J. Org. Chem. 2012, 77(1), 200.

[34] Feng, X. W.; Li, C.; Wang, N.; Li, K.; Zhang, W. W.; Wang, Z.; Yu, X. Q. Green Chem. 2009, 11(12), 1933.

[35] Majumder, A. B.; Ramesh, N. G.; Gupta, M. N. Tetrahedron Lett. 2009, 50(37), 5190.

[36] Yao, S. P.; Lu, D. S.; Wu, Q.; Cai, Y.; Xu, S. H.; Lin, X. F. Chem. Commun. 2004, (17), 20067.

[37] Wu, M. Y.; Li, K.; He, T.; Feng, X. W.; Wang, N.; Wang, X. Y.; Yu, X. Q. Tetrahedron 2011, 67(14), 2681.

[38] Chai, S. J.; Lai, Y. F.; Xu, J. C.; Zheng, H.; Zhu, Q.; Zhang, P. F. Adv. Synth. Catal. 2011, 353(2 3), 371.

[39] Xu, J. C.; Li, W. M.; Zheng, H.; Lai, Y. F.; Zhang, P. F. Tetrahedron 2011, 67(49), 9582.

[40] Li, K.; He, T.; Li, C.; Feng, X. W.; Wang, N.; Yu, X. Q. Green Chem. 2009, 11(6), 777.

[41] He, T.; Li, K.; Wu, M. Y.; Feng, X. W.; Wang, N.; Wang, H. Y.; Li, C.; Yu, X. Q. J. Mol. Catal., B: Enzym. 2010, 67(3 4), 189.

[42] Chai, S. J.; Lai, Y. F.; Zheng, H.; Zhang, P. F. Helv. Chim. Acta 2010, 93(11), 2231.

[43] Xue, Y.; Li, L. P.; He, Y. H.; Guan, Z. Sci. Rreports 2012, 2.

[44] Wang, J. L.; Liu, B. K.; Yin, C.; Wu, Q.; Lin, X. F. Tetrahedron 2011, 6(14), 2689. 